\title{
Coexistence of Charcot-Marie-Tooth 1A and nondystrophic myotonia due to PMP22 duplication and SCN4A pathogenic variants: a case report
}

Haitian Nan ${ }^{1 \dagger}$, Yunqing $\mathrm{Wu}^{2 \dagger}$, Shilei Cui ${ }^{2}$, Houliang Sun ${ }^{2}$, Jiawei Wang ${ }^{2}$, Ying Li ${ }^{3}$, Lingchao Meng ${ }^{3}$, Takamura Nagasaka ${ }^{4}$ and Liyong $\mathrm{Wu}^{1^{*}}$ (D)

\begin{abstract}
Background: Charcot-Marie-Tooth disease (CMT) is a genetically heterogeneous hereditary neuropathy, and CMT1A is the most common form; it is caused by a duplication of the peripheral myelin protein 22 (PMP22) gene. Mutations in the transient sodium channel Nav1.4 alpha subunit (SCN4A) gene underlie a diverse group of dominantly inherited nondystrophic myotonias that run the spectrum from subclinical myopathy to severe muscle stiffness, disabling weakness, or frank episodes of paralysis.
\end{abstract}

Case presentation: We describe a Chinese family affected by both CMT1A and myotonia with concomitant alterations in both the PMP22 and SCN4A genes. In this family, the affected proband inherited the disease from his father in an autosomal dominant manner. Genetic analysis confirmed duplication of the PMP22 gene and a missense c.3917G > C (p. Gly1306Ala) mutation in SCN4A in both the proband and his father. The clinical phenotype in the proband showed the combined involvement of skeletal muscle and peripheral nerves. Electromyography showed myopathic changes, including myotonic discharges. MRI revealed the concurrence of neurogenic and myogenic changes in the lower leg muscles. Sural nerve biopsies revealed a chronic demyelinating and remyelinating process with onion bulb formations in the proband. The proband's father presented with confirmed subclinical myopathy, very mild distal atrophy and proximal hypertrophy of the lower leg muscles, pes cavus, and areflexia.

Conclusion: This study reports the coexistence of PMP22 duplication and SCN4A mutation. The presenting features in this family suggested that both neuropathy and myopathy were inherited in an autosomal dominant manner. The proband had a typical phenotype of sodium channel myotonia (SCM) and CMT1A. However, his father with the same mutations presented a much milder clinical phenotype. Our study might expand the genetic and phenotypic spectra of neuromuscular disorders with concomitant mutations.

Keywords: Charcot-Marie-Tooth disease, Nondystrophic myotonia, PMP22, SCN4A, Case report

*Correspondence: wmywly@hotmail.com

${ }^{\dagger}$ Haitian Nan and Yunqing Wu contributed equally to this work.

${ }^{1}$ Department of Neurology, Xuanwu Hospital, Capital Medical University,

Beijing, China

Full list of author information is available at the end of the article

\section{Background}

Charcot-Marie-Tooth disease (CMT) comprises the most common group of disorders of the peripheral nervous system and is clinically and genetically heterogeneous. The most common CMT type is CMT1A, which is due to a dominantly inherited 1.5 megabase duplication 
on the short arm of chromosome 17 at locus p11.2 [1]. This duplicated segment contains the PMP22 gene that encodes peripheral myelin protein 22 .

Nondystrophic myotonias (NDMs) are a group of hereditary muscle diseases characterized by myotonia, muscle stiffness, and a nondystrophic phenotype, which are caused predominantly by mutations in CLCN1 or SCN4A [2]. SCN4A myotonias are clinically classified into three subgroups: sodium channel myotonia (SCM), paramyotonia congenita (PMC), and hyperkalemic periodic paralysis (Hyper PP) [3].

Because the estimated prevalence of SCM is approximately 0.06 per 100,000 population [4] and the calculated prevalence of CMT1A is in the range of $26: 100,000$ to $8: 100,000$ [5], the theoretical chance of inheriting both diseases is extremely low. Herein, we describe a Chinese family with a 1.5 megabase duplication including $P M P 22$ and a heterozygous mutation G1306A in SCN4A. The proband had a typical phenotype of both SCM and CMT1A. However, his father with the same mutations presented a much milder clinical phenotype.

\section{Case presentation \\ Clinical study}

The pedigree is shown in Fig. 1 A. The proband (Fig. 1 A, III-1), a 29-year-old male, was the only child of unrelated parents. His development was normal. As a child, he kept up with his peers physically. However, he displayed high-arched feet since adolescence. He began experiencing muscle stiffness in both hands and legs from his late teens, mostly in the mornings. Stiffness could also appear after a long exercise. Progressive leg weakness with drop feet and a steppage gait began insidiously during his twenties. At 28 years of age, his leg weakness worsened, and he developed numbness in the distal upper and lower extremities. At age 29, distal weakness of the hands affecting the dorsal interossei with reduced abduction in the metacarpophalangeal joints and weakness in thumb abduction occurred, and he presented hand deformities characterized by curvature of the fingers (Fig. $1 \mathrm{~B}$ ). The patient reported hypoesthesia of fingers and toes with poor finger control, and he had difficulties buttoning up his clothes or using chopsticks. Myotonia could be induced by hand grip and was relieved by tapping or massage for $4-5 \mathrm{~s}$. Symptoms were not exacerbated by cold exposure. There was no typical warm-up phenomenon, and he never experienced myalgia or paralytic attacks. Currently, he is still ambulatory with crutches.

Neurological examination revealed a steppage gait, drop feet, pes cavus deformity, hammertoes, claw hand, grip myotonia, and symmetric distal upper and lower extremity weakness. The bilateral proximal muscle strength of the upper extremities and lower extremities was normal. The strength in distal muscles of the upper extremities, including the wrist flexors and extensors, was $3 / 5$ on the medical research council scale (MRC). The strength in the intrinsic hand muscles, including the finger flexors, the first dorsal interosseous, the abductor digiti minimi, and the abductor pollicis brevis, was MRC grade $2 / 5$. The muscle strength levels of the ankle and toe extensors were MRC grades $3 / 5$ and $2 / 5$, respectively, while ankle dorsiflexion was completely restricted on both sides. There was mild atrophy of the distal muscles of the hands, particularly of the thenar eminence (Fig. 1 B). Subtle wasting of the thigh musculature was noted, whereas very mild calf hypertrophy was also identified (Fig. 1 C). He had difficulty with tandem gait and had decreased sensitivity to vibration and pain in a stockingglove distribution involving the upper limbs to the level of the elbows and the lower limbs to the mid-calf. Deep tendon reflexes were absent, and plantar reflexes were flexor. There were no abnormalities in the cranial nerves or his coordination. Stork legs, scoliosis, lid lag, dysphagia, dysarthria, myokymia, fasciculations, joint malformations, or pyramidal tract signs were not present.

His creatine kinase (CK) levels were $224 \mathrm{U} / \mathrm{L}$ and $386 \mathrm{U} / \mathrm{L}$ on repeated measurements (reference range, 25-240 U/L). The liver function tests, albumin, serum potassium levels, thyroid function, and autoantibody levels (including anti-Jo1 and anti-acetylcholine receptor) were within normal ranges. His brain and spinal magnetic resonance imaging (MRI) results were normal. His echocardiogram was normal without signs of hypertrophy and with normal relaxation time. Muscle MRI of the proband showed the concurrence of both fatty degeneration and very mild hypertrophy in the lower leg muscles (Fig. 1 D).

Needle electromyography (EMG) was performed in the right abductor policis brevis, the left extensor digitorum muscle, the left biceps muscle, the left gastrocnemius muscle, the right vastus medialis, and the right tibialis anterior muscle. All muscles demonstrated typical runs of myotonic discharges (Fig. $1 \mathrm{E}$ ). Unfortunately, the patient rejected exerting strength with pain, so we could only record insertion activity. We observed some motor unit action potentials (MUAPs), with long duration relative to modest amplitude (amplitude $0.2-0.9 \mu \mathrm{V}$, duration 3-7 ms) with decreased interference, in the extensor digitorum communis, which appeared in a short time before myotonic discharge (an additional movie file shows this in more detail [see Additional file 1]). MUPs may reveal the coexistence of a muscle disorder as well as neuropathy. Motor nerve conduction velocities were markedly decreased (median nerve, $15.5 \mathrm{~m} / \mathrm{s}$; ulnar nerve, $17 \mathrm{~m} / \mathrm{s}$; tibial nerve, $13.5 \mathrm{~m} / \mathrm{s}$; peroneal nerve, $16.8 \mathrm{~m} / \mathrm{s}$ ). Distal motor latencies were prolonged (median nerve, $15.4 \mathrm{~ms}$; 


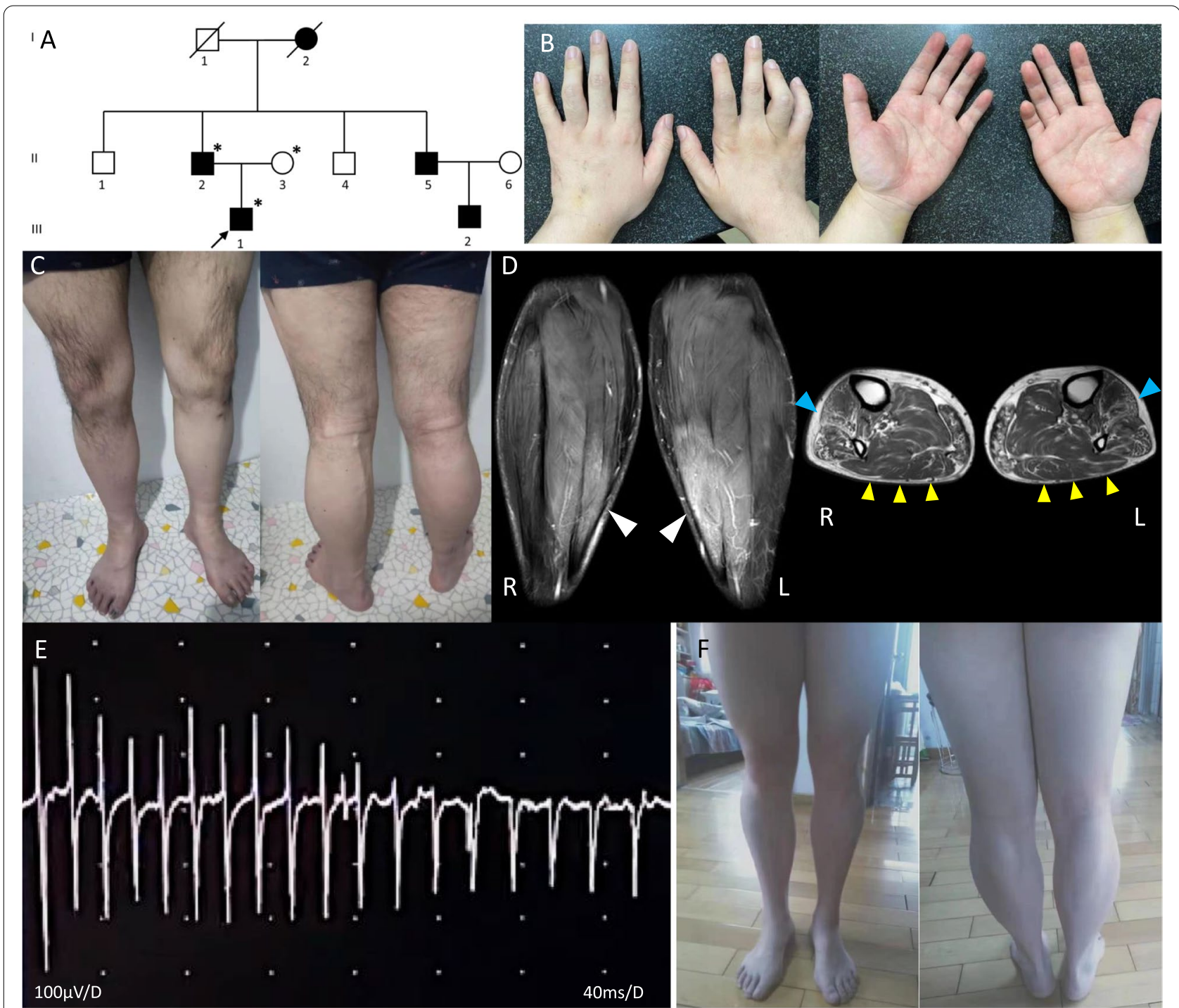

Fig. 1 A Pedigree of the present family. The proband is indicated (arrow). Individuals evaluated both clinically and genetically in this study are denoted by asterisks. B Serial photographs of the proband taken at age 29. The proband presented hand deformities characterized by curvature of the fingers. There was mild atrophy of the distal muscles of the hands, particularly of the thenar eminence. C Appearance of the legs of the proband. There was mild quadriceps wasting and simultaneous mild calf hypertrophy. D Fat-saturated MRI of the lower legs in the coronal plane and T2-weighted image in the axial plane of the lower third of the calves of the proband. High-intensity regions manifesting as neurogenic changes can be easily observed in the bilateral triceps surae muscles, with proximal dominant hypertrophy in the coronal plane (white arrowhead). There was moderate fatty atrophy involving bilateral tibialis anterior muscles in the axial plane (blue arrowhead), which predominated on the right side. Conversely, there was very slight hypertrophy in the posterior compartment muscles (yellow arrowhead), including the soleus, gastrocnemius, and tibialis posterior. $R$ and $L$ indicate right and left. E Electrophysiologic findings in the proband recorded from the left extensor digitorum communis. The insertion activity of needle electromyography at rest shows myotonic discharges (timebase set to $40 \mathrm{~ms} /$ division). $\mathbf{F}$ Appearance of the legs of the proband's father at age 57. Pes cavus is pronounced. Mild proximal hypertrophy and distal atrophy of bilateral lower leg muscles were observed

ulnar nerve, $12 \mathrm{~ms}$; tibial nerve, $12.2 \mathrm{~ms}$; peroneal nerve, $8.16 \mathrm{~ms}$ ), and compound muscle action potential (CMAP) amplitudes were markedly decreased (median nerve: $0.43 \mathrm{mV}$, ulnar nerve: $0.21 \mathrm{mV}$, tibial nerve: $0.074 \mathrm{mV}$, peroneal nerve: $0.11 \mathrm{mV}$ ). Sensory nerve action potentials (SNAPs) were not elicited in the upper or lower extremities. Nerve conduction study results were consistent with motor and sensory demyelinating neuropathy (Table 1). Decreased CMAP amplitude could be a reflection of the reduced muscle fiber volume due to severe fiber loss.

The proband's father (Fig. 1 A, II-2), a 57-year-old male, presented pes cavus feet and no other related manifestations. His deep tendon reflexes were absent. The remainder of the neurological examination was 
Table 1 Electrophysiologic studies of the proband reported in this study

\begin{tabular}{|c|c|c|}
\hline & Proband & Normal range \\
\hline \multicolumn{3}{|l|}{ Median nerves } \\
\hline DML (ms) & 15.4 & $<4.4$ \\
\hline $\mathrm{MCV}(\mathrm{m} / \mathrm{s})$ & 15.5 & $>49$ \\
\hline Proximal CMAP (mV) & 0.43 & $>4$ \\
\hline \multicolumn{3}{|l|}{ Ulnar nerves } \\
\hline DML (ms) & 12 & $<3.3$ \\
\hline $\mathrm{MCV}(\mathrm{m} / \mathrm{s})$ & 17.0 & $>49$ \\
\hline Proximal CMAP (mV) & 0.21 & $>6$ \\
\hline $\mathrm{SCV}(\mathrm{m} / \mathrm{s})$ & 25.3 & $>47$ \\
\hline $\operatorname{SNAP}\left({ }_{\mu} \mathrm{V}\right)$ & - & $>3$ \\
\hline $\mathrm{DML}(\mathrm{ms})$ & 4.75 & \\
\hline \multicolumn{3}{|l|}{ Peroneal nerves } \\
\hline $\mathrm{DML}(\mathrm{ms})$ & 8.16 & $<5.8$ \\
\hline $\mathrm{MCV}(\mathrm{m} / \mathrm{s})$ & 16.8 & $>41$ \\
\hline Proximal CMAP (mV) & 0.11 & $>4$ \\
\hline \multicolumn{3}{|l|}{ Tibial nerves } \\
\hline $\mathrm{DML}(\mathrm{ms})$ & 12.2 & $<5.8$ \\
\hline $\mathrm{MCV}(\mathrm{m} / \mathrm{s})$ & 13.5 & $>41$ \\
\hline Proximal CMAP (mV) & 0.074 & $>4$ \\
\hline
\end{tabular}

DML distal motor latency, MCV motor conduction velocity, CMAP compound muscle action potential, SCV sensory conduction velocity, SNAP sensory nerve action potential

normal, including sensory and manual muscle testing. He showed very mild distal atrophy and proximal hypertrophy of the lower leg muscles (Fig. 1 F). Despite not having symptoms related to myotonia, myotonic discharges were confirmed in the left biceps muscle on needle EMG examination (see Additional file 2). Unfortunately, a nerve conduction study of the proband's father was not performed, and we could not obtain more information.

The proband's deceased paternal grandmother (Fig. 1 A, I-2), his paternal uncle (Fig. 1 A, II-5), and his paternal uncle's son (Fig. 1 A, III-2) also had pes cavus, but they have not been reviewed at our center. They did not report any neurological symptoms. No other family members are known to be affected in this pedigree. The proband's mother (Fig. 1 A, II-3) did not show any neurological abnormalities.

\section{Genetic study}

We carried out whole-exome sequencing of genomic DNA from the proband. Genomic DNA was isolated from peripheral blood leukocytes using standard methods. Exome capture was performed with a SureSelect Human All Exon V6+UTR (89 Mb) Kit (Agilent Technologies, Santa Clara, CA, USA). Paired-end sequencing was carried out on a HiSeq2500 (Illumina, San Diego, CA, USA) using a HiSeq SBS Kit V4 (Illumina), which generated 100-bp reads. The reference databases utilized included hg19 (GRCh37) (http://genome.ucsc. edu), HGMD (https://portal.biobase-international. com), GnomAD (http://gnomad.broadinstitute.org), and dbSNP (https://www.ncbi.nlm.nih.gov/snp/). We examined variants of genes known to be responsible for neuromuscular diseases such as CMT or myotonia (https:// neuromuscular.wustl.edu/). Through this analysis, we identified a c.3917G >C (p. Gly1306Ala) mutation in exon 22 of the SCN4A gene (NM_000334) and ruled out point mutations in other causative genes for neuromuscular diseases. We then carried out Sanger sequencing of genomic DNA from the proband (Fig. $1 \mathrm{~A}, \mathrm{III}-1$ ), the proband's father (Fig. $1 \mathrm{~A}, \mathrm{II}-2$ ), and the proband's mother (Fig. 1 A, II-3). We reconfirmed the c.3917G $>$ C (p. Gly1306Ala) mutation in exon 22 of the $S C N 4 A$ gene, which was in a heterozygous state in the proband (Fig. 2 A) and his father (Fig. 2 C). However, this mutation was not detected in the proband's mother, who was without symptoms (Fig. 2 E).

Furthermore, we used our in-house script aimed to find copy number variants (CNVs) and genotype the copy numbers of duplicated genes by analyzing exome sequencing data. We detected a probable duplicated segment on the short arm of chromosome 17 at locus p11.2, which contains the PMP22 gene in the proband. Multiplex ligation-dependent probe analysis (MLPA) was then used to verify the duplications in the PMP22 gene. MLPA was performed using the MLPA kit (SALSA MLPA probemix P405-A1 CMT; MRC Holland, Amsterdam, Netherlands) according to the manufacturer's protocol. Cutoff values for duplication and deletion were set as $>1.2$ and $<0.7$, respectively [6]. MLPA studies revealed the presence of a heterozygous $1.5 \mathrm{Mb}$ duplication in 17p11.2-p12, including the coding regions of PMP22 in the proband (Fig. $2 \mathrm{~B}$ ) and the proband's father (Fig. $2 \mathrm{D}$ ). However, this duplication in $P M P 22$ was negative in the proband's mother (Fig. 2 F). We thus demonstrated that both the duplication in PMP22 and the p.G1306A mutation in SCN4A in the proband were inherited from his father.

\section{Neuropathological study}

Sural nerve biopsy was performed in the proband with both SCN4A and PMP22 alterations. The samples were initially fixed in $2.5 \%$ glutaraldehyde, followed by $1 \%$ buffered osmium tetroxide, and then dehydrated in ascending grades of ethanol and embedded in Epon. Transverse semithin sections were stained with toluidine blue. Sural nerve biopsies revealed a severe loss of myelinated fibers. There was some axonal degeneration and regenerating clusters of myelinated fibers. Onion bulb formation was frequent (Fig. 2 G). Unfortunately, we failed to obtain 


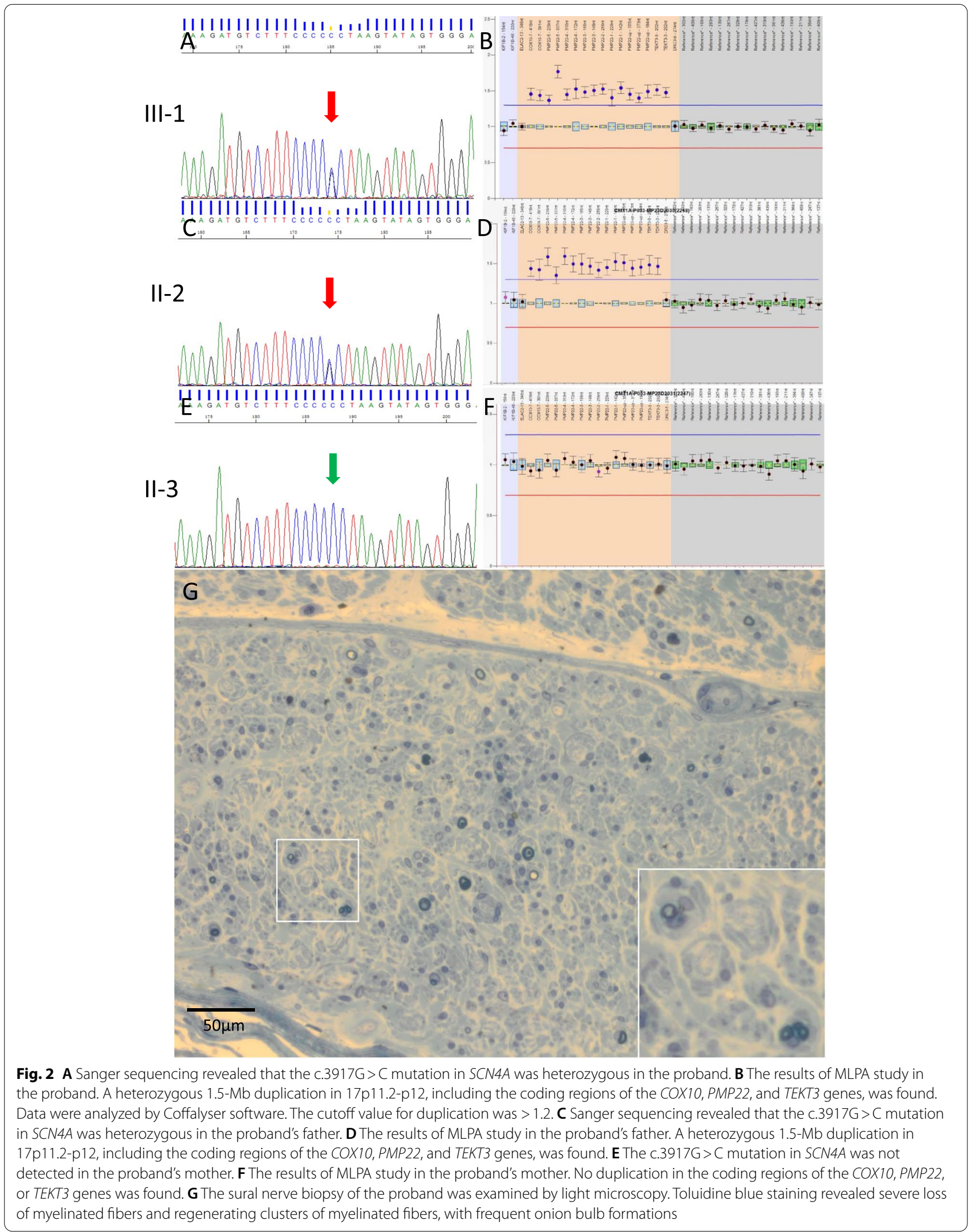


Table 2 Clinical features of the proband and his father with PMP22 and SCN4A mutations

\begin{tabular}{|c|c|c|c|}
\hline \multicolumn{2}{|l|}{ Patient } & \multirow{2}{*}{$\begin{array}{l}\text { Proband } \\
\text { c.3917G>C p. Gly1306Ala }\end{array}$} & \multirow{2}{*}{$\begin{array}{l}\text { Proband's father } \\
\text { c.3917G > C p. Gly1306Ala }\end{array}$} \\
\hline SCN4A gene studies & & & \\
\hline PMP22 gene studies & & Duplication in PMP22 & Duplication in PMP22 \\
\hline Sex & & Male & Male \\
\hline Age (yrs) & & 29 & 57 \\
\hline Age at Onset & & Adolescence & Unknown \\
\hline First symptom at onset & & High-arched feet & High-arched feet \\
\hline Muscular Stiffness & & Yes, mostly in the mornings or after long exercises & No \\
\hline Muscle pain & & No & No \\
\hline \multirow[t]{2}{*}{ Muscle weakness } & Upper limb & Wrist muscles $3 / 5$, intrinsic hand muscles $2 / 5$ & No \\
\hline & Lower limb & $\begin{array}{l}\text { Ankle and toe extensors }<3 / 5 \text {, ankle dorsiflexion } \\
\text { restricted }\end{array}$ & No \\
\hline Muscle atrophy & & $\begin{array}{l}\text { Mild atrophy of the distal muscles of the hands, } \\
\text { subtle wasting of thigh musculature }\end{array}$ & Very mild distal atrophy of the lower leg muscles \\
\hline Muscle hypertrophy & & Mild calves hypertrophy & $\begin{array}{l}\text { Very mild proximal hypertrophy of the lower leg } \\
\text { muscles }\end{array}$ \\
\hline Warm up phenomenon & & No & Not Applicable \\
\hline Handgrip myotonia & & Yes & No \\
\hline Myotonia worsened by cold & & No & Not Applicable \\
\hline Myotonia distribution & & Hands, arms, legs & Not Applicable \\
\hline Electromyography & & Positive for generalized typical myotonic discharges & $\begin{array}{l}\text { Myotonic discharges were confirmed in the left } \\
\text { biceps muscle }\end{array}$ \\
\hline CK(U/L) & & $224 \mathrm{U} / \mathrm{L}, 386 \mathrm{U} / \mathrm{L}$ & Not examined \\
\hline Pes cavus & & Yes & Yes \\
\hline Sensory loss & & $\begin{array}{l}\text { A stocking-glove distribution involving the upper } \\
\text { limbs to the level of the elbows and the lower limbs } \\
\text { to the mid-calf }\end{array}$ & No \\
\hline Deep tendon reflexes & & Absent & Absent \\
\hline Plantar response & & Flexor & Flexor \\
\hline Sural nerve biopsy & & Severe demyelinating neuropathy & Not examined \\
\hline CMTNS & & Severe (26) & Mild \\
\hline Recurrent flaccid paralysis & & No & No \\
\hline Scoliosis & & No & No \\
\hline
\end{tabular}

Abbreviations: CMTNS CMT neuropathy score

muscle tissues during the biopsy due to severe fatty degeneration of the distal lower leg muscles.

\section{Discussion and conclusions}

The occurrence of two genetically unlinked disorders in a patient or a pedigree is rare, but studies include descriptions of a boy with myotonia congenita $(\mathrm{MC})$ and CMT1A [7], patients affected with both MC and SCN4Amyotonias [8-10], and a family with a mutation identified in $M P Z$ in two patients with CMT and a mutation identified in SCN4A in 4 family members with Hyper PP [11]. To our knowledge, our report represents the first case of cosegregation of SCN4A myotonias and CMT1A.

The G1306A missense variant in the SCN4A gene has been reported multiple times in association with myotonia. Nav1.4-G1306 is localized in the III-IV intracellular loop of the voltage-gated sodium channel of skeletal muscle, which contains the fast inactivation particle. At this position, there are three observed genotypes to be distinguished, of which G1306V and G1306E often lead to more severe phenotypes [12], while G1306A mostly leads to the least severe, a mild form of SCM [13], characterized by nondystrophic, generalized myotonia with daily fluctuations and muscle stiffness after exercise without substantial cold sensitivity [14-18]. Muscle pain is not a frequent symptom [12], while CK values are frequently elevated in such patients, with average elevations of 2-3-fold, indicating mild myolysis [12]. In our proband, myotonia was also observed both clinically and electrophysiologically. He had handgrip myotonia, 
postexercise myotonia, and mild muscle hypertrophy. He also showed myotonic discharges on electromyography. His CK levels were either normal or mildly elevated. These findings suggest an SCM phenotype, thus supporting the pathogenic role of the $S C N 4 A$ mutation. Moreover, muscle weakness and atrophy, sensory disturbance, and abnormal tendon reflexes were also detected, which phenotypically fit into CMT1A.

Mutations of the PMP22 gene in combination with other genetic variants have been previously reported to cause a more severe phenotype than the one expected by $P M P 22$ mutation alone [19]. Interestingly, the proband's father displayed a milder phenotype despite the presence of both $P M P 22$ and SCN $4 A$ variations. $\mathrm{He}$ was not aware of any neurologic or myogenic symptoms, but myotonia was observed in the electrophysiological examination. The clinical features of the proband and his father are summarized in Table 2 . There is an anticipation phenomenon in CMT1A patients [20]. Moreover, the penetrance of SCN4A myotonias is high, particularly in males. In this family, apparent genetic anticipation of peripheral neuropathy was also observed. The CMT1A symptoms of muscle weakness, sensory disturbance, and steppage gait of the proband were absent in the proband's father. However, the proband had a relatively mild myotonia phenotype, so genetic anticipation of myotonia could not be concluded in this family. However, we cannot predict whether this condition in our proband could lead to more severe clinical impairment with age. It is of interest that the proband and his father presented different clinical phenotypes even though they had the same mutations. There might be some unknown genetic etiology or environmental modifiers underlying the phenotypes that could not be identified with our present methods. Further functional study may be warranted to identify the underlying cellular and molecular mechanisms for this disease.

At first, pure myotonia went unnoticed in the proband because of a preponderance of the more severe condition of peripheral neuropathy. It was not until the $S C N 4 A$ mutation was identified by WES analysis that we stepped back and recollected the medical data of the proband. Clinicians should consider routinely performing comprehensive genetic tests for patients with atypical symptoms, even in the case that one pathological mutation has already been identified.

In conclusion, we present a family with a combination of mutations in PMP22 and SCN4A, leading to the coexistence of two autosomal dominantly inherited disorders: CMT1A and SCM. The clinical phenotype in the proband showed the combined involvement of skeletal muscle and peripheral nerves. His father with the same mutations presented a much milder phenotype. This rare genetic coincidence might expand the genetic and phenotypic spectra of neuromuscular disorders with concomitant mutations.

\section{Abbreviations}

CMT: Charcot-Marie-Tooth disease; SCM: Sodium channel myotonia; NDM: Nondystrophic myotonia; PMC: Paramyotonia congenita; Hyper PP: Hyperkalemic periodic paralysis; MRC: Medical research council scale; MRI: Magnetic resonance imaging; EMG: Electromyography; CMAP: Compound muscle action potential; SNAP: Sensory nerve action potentials; CNV: Copy number variant; MLPA: Multiplex ligation-dependent probe analysis; PCR: Polymerase chain reaction; MC: Myotonia congenita; CK: Creatine kinase.

\section{Supplementary Information}

The online version contains supplementary material available at https://doi. org/10.1186/s12883-021-02538-5.

Additional file 1. EMG of the proband recorded from the left extensor digitorum communis showed complex repetitive discharges and myotonic discharges.

Additional file 2. EMG of the proband's father recorded from the left biceps muscle showed myotonic discharges.

Additional file 3.

\section{Acknowledgments}

We wish to thank the patient and his family for participation in this study.

\section{Authors' contributions}

$\mathrm{HN}$ : drafted the manuscript, performed the genetic analysis, and interpreted the genetic data. YW: mainly provided and analyzed the clinical data. LM and $Y L$ acquired and analyzed the pathological and neuroimaging data. TN: analyzed and interpreted the neuroimaging, electrophysiological, and pathological findings. SC, HS, and JW: acquisition and analysis of the clinical data. LW: conception of the work and manuscript revision. All authors read and approved the contents of the case report.

\section{Funding}

This work was supported by the Ministry of Science and Technology of China (2019YFC0118600), the National Natural Science Foundation of China (No. 81971011), and the Beijing Municipal Science and Technology Committee (D171100008217005, 7202060).

\section{Availability of data and materials}

All data generated or analyzed during this study are included in this published article.

\section{Declarations}

\section{Ethics approval and consent to participate}

The case report complies with the Helsinki Declaration, and approval was obtained from the Ethical Committee of Capital Medical University. Written informed consents were obtained from the proband and his parents.

\section{Consent for publication}

Written informed consents were obtained from the proband and his parents for publication of this case report including the pedigree diagram and any other associated images.

Competing interests

The authors declare that they have no conflict of interest. 


\section{Author details}

${ }^{1}$ Department of Neurology, Xuanwu Hospital, Capital Medical University, Beijing, China. ${ }^{2}$ Department of Neurology, Beijing Tongren Hospital, Capital Medical University, Beijing, China. ${ }^{3}$ Department of Neurology, Peking University First Hospital, Beijing, China. ${ }^{4}$ Department of Neurology, University of Yamanashi, 1110 Shimokato, Chuo-city, Yamanashi 409-3898, Japan.

Received: 14 July 2021 Accepted: 27 December 2021

Published online: 07 January 2022

\section{References}

1. Lupski JR, de Oca-Luna RM, Slaugenhaupt S, Pentao L, Guzzetta V, Trask BJ, et al. DNA duplication associated with Charcot-Marie-tooth disease type 1A. Cell. 1991;66(2):219-32. https://doi.org/10.1016/0092-8674(91) 90613-4.

2. Matthews E, Fialho D, Tan SV, Venance SL, Cannon SC, Sternberg D, et al. The non-dystrophic myotonias: molecular pathogenesis, diagnosis and treatment. Brain. 2010;133(Pt 1):9-22. https://doi.org/10.1093/brain/ awp294.

3. Heatwole CR, Moxley RT 3rd. The nondystrophic myotonias. Neurotherapeutics. 2007;4(2):238-51. https://doi.org/10.1016/j.nurt.2007.01.012.

4. Horga A, Raja Rayan DL, Matthews E, Sud R, Fialho D, Durran SC, et al. Prevalence study of genetically defined skeletal muscle channelopathies in England. Neurology. 2013;80(16):1472-5. https://doi.org/10.1212/WNL. Ob013e31828cf8d0.

5. van Paassen BW, van der Kooi AJ, van Spaendonck-Zwarts KY, Verhamme C, Baas F, de Visser M. PMP22 related neuropathies: Charcot-Marie-tooth disease type $1 \mathrm{~A}$ and hereditary neuropathy with liability to pressure palsies. Orphanet J Rare Dis. 2014;9:38. https://doi.org/10.1186/ 1750-1172-9-38.

6. Stuppia L, Antonucci I, Palka G, Gatta V. Use of the MLPA assay in the molecular diagnosis of gene copy number alterations in human genetic diseases. Int J Mol Sci. 2012;13(3):3245-76. https://doi.org/10.3390/ijms 1 3033245.

7. Ardissone A, Brugnoni R, Gandioli C, Milani M, Ciano C, Uziel G, et al. Double-trouble in pediatric neurology: myotonia congenita combined with charcot-marie-tooth disease type 1a. Muscle Nerve. 2014;50(1):1457. https://doi.org/10.1002/mus.24205.

8. Zhao C, Tang D, Huang H, Tang H, Yang Y, Yang M, et al. Myotonia congenita and periodic hypokalemia paralysis in a consanguineous marriage pedigree: coexistence of a novel CLCN1 mutation and an SCN4A mutation. PLoS One. 2020;15(5):e0233017. https://doi.org/10.1371/journ al.pone.0233017.

9. Kato H, Kokunai Y, Dalle C, Kubota T, Madokoro Y, Yuasa H, et al. A case of non-dystrophic myotonia with concomitant mutations in the SCN4A and CLCN1 genes. J Neurol Sci. 2016;369:254-8. https://doi.org/10.1016/j.jns. 2016.08.030.

10. Maggi L, Ravaglia S, Farinato A, Brugnoni R, Altamura C, Imbrici P, et al. Coexistence of CLCN1 and SCN4A mutations in one family suffering from myotonia. Neurogenetics. 2017;18(4):219-25. https://doi.org/10.1007/ s10048-017-0525-5.

11. Hisama FM. Familial periodic paralysis and Charcot-Marie-tooth disease in a 7-generation family. Arch Neurol. 2005;62(1):135-8. https://doi.org/10. 1001/archneur.62.1.135.

12. Torbergsen $T$, Jurkat-Rott $K$, Stalberg EV, Loseth S, Hodneo A, LehmannHorn F. Painful cramps and giant myotonic discharges in a family with the Nav1.4-G1306A mutation. Muscle Nerve. 2015;52(4):680-3. https://doi. org/10.1002/mus.24672

13. Ricker K, Lehmann-Horn F, Moxley RT 3rd. Myotonia fluctuans. Arch Neurol. 1990;47(3):268-72. https://doi.org/10.1001/archneur.1990.00530 030034012.

14. Lerche H, Heine R, Pika U, George AL Jr, Mitrovic N, Browatzki M, et al. Human sodium channel myotonia: slowed channel inactivation due to substitutions for a glycine within the III-IV linker. J Physiol. 1993;470:1322. https://doi.org/10.1113/jphysiol.1993.sp019843.

15. Ricker K, Moxley RT 3rd, Heine R, Lehmann-Horn F, Myotonia fluctuans. A third type of muscle sodium channel disease. Arch Neurol. 1994;51(11):1095-102. https://doi.org/10.1001/archneur.1994.00540 230033009
16. Morrow JM, Matthews E, Raja Rayan DL, Fischmann A, Sinclair CD, Reilly $M M$, et al. Muscle MRI reveals distinct abnormalities in genetically proven non-dystrophic myotonias. Neuromuscul Disord. 2013;23(8):637-46. https://doi.org/10.1016/j.nmd.2013.05.001.

17. Trivedi JR, Bundy B, Statland J, Salajegheh M, Rayan DR, Venance SL, et al. Non-dystrophic myotonia: prospective study of objective and patient reported outcomes. Brain. 2013;136(Pt 7):2189-200. https://doi.org/10. 1093/brain/awt133.

18. Meyer AP, Roggenbuck J, LoRusso S, Kissel J, Smith RM, Kline D, et al. Genotype-phenotype correlations and characterization of medication use in inherited Myotonic disorders. Front Neurol. 2020;11:593. https:// doi.org/10.3389/fneur.2020.00593.

19. Hodapp JA, Carter GT, Lipe HP, Michelson SJ, Kraft GH, Bird TD. Double trouble in hereditary neuropathy: concomitant mutations in the PMP22 gene and another gene produce novel phenotypes. Arch Neurol. 2006;63(1):112-7. https://doi.org/10.1001/archneur.63.1.112.

20. Steiner I, Gotkine M, Steiner-Birmanns B, Biran I, Silverstein S, Abeliovich $D$, et al. Increased severity over generations of Charcot-Marie-tooth disease type 1A. J Neurol. 2008;255(6):813-9. https://doi.org/10.1007/ s00415-008-0693-1.

\section{Publisher's Note}

Springer Nature remains neutral with regard to jurisdictional claims in published maps and institutional affiliations.

Ready to submit your research? Choose BMC and benefit from:

- fast, convenient online submission

- thorough peer review by experienced researchers in your field

- rapid publication on acceptance

- support for research data, including large and complex data types

- gold Open Access which fosters wider collaboration and increased citations

- maximum visibility for your research: over $100 \mathrm{M}$ website views per year

At BMC, research is always in progress.

Learn more biomedcentral.com/submissions 